Ma et al., Afr J Tradit Complement Altern Med. (2014) 11(2):301-314

\title{
AN ASSESSMENT OF TRADITIONAL UIGHUR MEDICINE IN CURRENT XINJIANG REGION (CHINA)
}

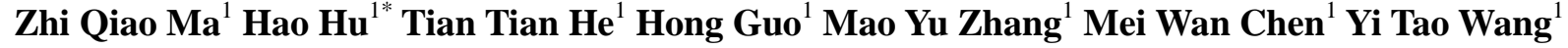

\author{
${ }^{1}$ State Key Laboratory of Quality Research in Chinese Medicine, Institute of Chinese Medical Sciences, University of \\ Macau, Macao \\ *Email: haohu@umac.mo
}

\begin{abstract}
Background: The main objectives of this study were to assess the current research and development of traditional Uighur medicine in Xinjiang (China), and to evaluate the promising pharmacological products of traditional Uighur medicine for further studies.

Materials and Methods: Traditional Uighur medicine data of medicine registry, patent, and academic publications was collected and analyzed. Results: Data showed that, among the registered and studied traditional Uighur medicine, the main therapeutic areas of traditional Uighur medicine focused on skin disease, urogenital disease, rheumatism and digestive system disease. The representative traditional Uighur patent medicine included the following: BaixuanXiatare Tablets, Kaliziran Tincture and Vernoniaanthelmintica Injection (Psoriasis and vitiligo); Xi-payimazibiziLiquid (prostatitis); KursiKaknaq (urinary tract infection); Tongzhisurunjiang Capsules (anti-rheumatism medicine); HuganBuzure Granules (digestive system disease). Moreover, ten Uighur herbs were widely used, including: ResinaScammoniae, Folium FumicisDentati, HerbaDracocephali, Semen AmygdaliDulcis, HerbaChamomillae, FructusPimpinellaeanisi, Cortex Foeniculi, FructusVernoniae, FructusApii, and Radix AnacycliPyrethri.

Conclusion: This study concluded by indicating that traditional Uighur medicine with excellent curative effect should be screened in details for their phytochemical properties and pharmacological activity to discover new bioactive constituents.
\end{abstract}

Key words: Traditional Uighur medicine, Traditional Uighur patent medicine, Uighur herb

\section{Introduction}

Traditional Uighur medicine (TUM) is one of the most important traditional medicines in Central Asia, which is rooted in the ancient Uighur medicine theory and has a history of more than 2,500 years. Currently, TUM is mainly applied over Central Asia, North Africa and South Europe (Wang et al., 2005). The traditional theory of Uighur medicine regards that the nature is constituted by four substances: fire, air, water, and earth (Amat et al., 2009; Geng, 2006). Built upon this theory, TUM in Xinjiang Province (China) has also combined the essence of other medical theories, such as traditional Chinese medicine, ancient Greece medicine, Egyptian medicine, Arabian medicine, and Indian medicine. It has formulated a profound theoretical system, which is composed of the four temperaments (hot, cold, moist, and dry) and the four body fluids (Kan (blood), Belghem (phlegm), Sapra (yellow bile), and Savda (black bile) (Upur et al., 2011). The theory of body fluids came from the ancient Unani medicine theory, the humorism. Based on the Humorae theory, TUM has emphasized the balance status of four temperaments and that conditioning of the body fluids has become the main therapeutic principles (Upur et al., 2011).

Based on its complete medical theory system, TUM in Xinjiang has accumulated rich clinical experiences in the past. In treating intractable diseases, TUM has shown distinguished medical methods and unique therapeutic effect. As a traditional ethnic medicine, TUM has made great contribution to not only the health care in Xinjiang but also the medical treatment around China. Moreover, it has benefited the people in the other countries of Central Asia, with their intimate collections of religious beliefs and language (see Figure 1).

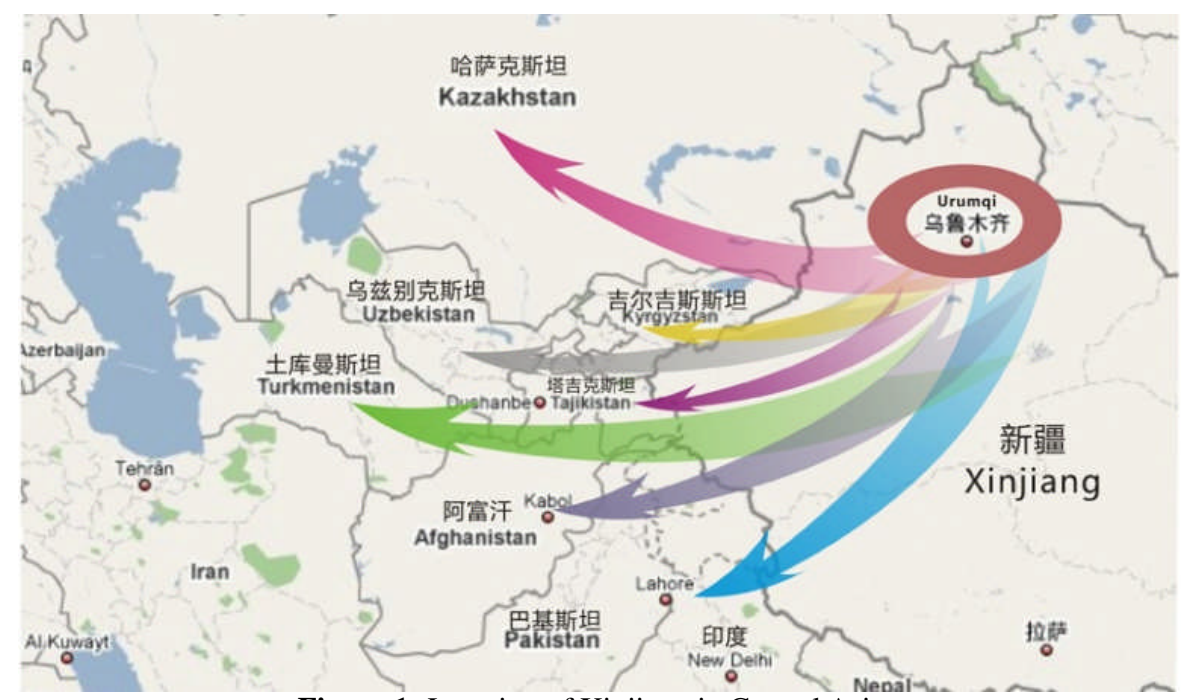

Figure 1: Location of Xinjiang in Central Asian

Despite its special medical contribution to patients, TUM in Xinjiang has not received the attention it deserves in modern research and development. Thus, this study aims at assessing the current research and development of Uighur medicine in Xinjiang thorough studying its registry, patent, and publications, hoping to provide inspiring knowledge about new pharmacological products of Uighur medicine. 
http://dx.doi.org/10.4314/ajtcam.v11i2.13

\section{Materials and Methods \\ Data source and collection}

To provide comprehensive analysis of TUM in Xinjiang, this study targeted not only traditional Uighur patent medicine (TUPM) but also Uighur herbs. TUPM are prescription preparations that follow the traditional Uighur medicine theory, which have been applied in medical treatment historically. Data of registry, patent and academic publication of TUM in Xinjiang was identified as main data types. All the data was searched and obtained from specific databases. Because the research of TUM is still at a primary stage and most of researches are from China, this study mainly focused on databases in China.

Firstly, the registry database of CFDA (China Food and Drug Administration) was used to search and screen the registered TUPM. The standard names of these TUPM were adopted from the Drug Standard of Ministry of Health of the People's Republic of China (Uighur Medicine Part), which was edited by The Ministry of Health of the People's Republic of China as a legal basis for TUM's manufacturing, sales, usage, and administration.

Secondly, the China Medicine Patent Database in China Intellectual Property Right Net (CNIPR) was used to search patents of TUPM and Uighur herbs. TUPM's name and formulation were searched by "ABSTRACT" in the database to identify target patents. For Uighur herbs, only herb name was used.

Thirdly, the academic publication data of TUPM and Uighur herbs were retrieved in the China National Knowledge Internet (CNKI). Both TUPM and Uighur herb were searched by name, with retrieval strategy as "SU= a medicine's name".

\section{Data analysis}

Data analysis was conducted in sequence. Firstly, we analyzed the information of registered TUPM, mainly focusing on therapeutic area, dosage and formulation. Secondly, patents and academic publications of TUPM were analyzed. For patents, analysis focused on patent's name, type and therapeutic area; for academic publications, analysis concentrated on paper quantity and research content. Thirdly, the ten Uighur herbs mostly used in TUPM were analyzed by similar analysis strategy of TUPM.

\section{Results \\ Registry of TUPM}

Data showed that 43 TUPMs listed in the Drug Standard of Ministry of Health of the People's Republic of China (Uighur Medicine Part, 2000 edition) had got official approval (see Table 1). For therapeutic areas, these TUPMs were mainly used for treating skin, urogenital system and digestive system diseases. Among these registered TUPMs, there are11 TUPMs treating skin disease, including Compound Siyadan Tincture, Qubaimaribairesi Pills, BaixuanXiatare Tablets, CompoundMuni Ziqi Granules, QubaiBabuqi Tablets, Compound Vernoniaanthelmintica Pills, Kaliziran Tincture, Vernoniaanthelmintica Injection, Suziafu Tablets, and Yangxindawayimixikemi Unguent. And 10 TUPMs are related to treat genitourinary system diseases, including Qiangshenluobofusaiheli Unguent, Wenshensulafu Tablets, Gujingmaisiha Tablets, Xi-payimazibizi Liquid, KursiKaknaq, Yanxiaodinaer Syrup, QingreKasen Granules, Luobufukebiri Tablets, Yimusake Tablets, and Jinsuokunduer Tablets. Additionally, 7 TUPMs are about treatment of digestive system diseases, including Mamuran antidiarrheal $l$ Capsules, QizilGulqent, Xipayi mouth rinse, Rose Oral Liquid, Tongzhiaitilefeilisana Tablets, HuganBuzure Granules, Sanhanyao Tea, and Compound Gaoziban Tablets.

Categorized through dosage, the approved TUPMs include about 10 different dosage forms, representatives as 15 in tablets, 7 in granules and 5 in mixture, taking the proportion $34.9 \%, 16.3 \%$ and $11.6 \%$ respectively. The only one TUPM injection is Vernonia anthelmintica Injection. Only 8 TUPMs were approved with traditional dosage forms of Uighur medicine, such as liniment, tincture and soft extract. Simultaneously, 20 TUPMs were approved as over-the-counter medicine, with the proportion $46.5 \%$ of the total.

Table 1: TUPM approved by CFDA (By therapy through ranking)

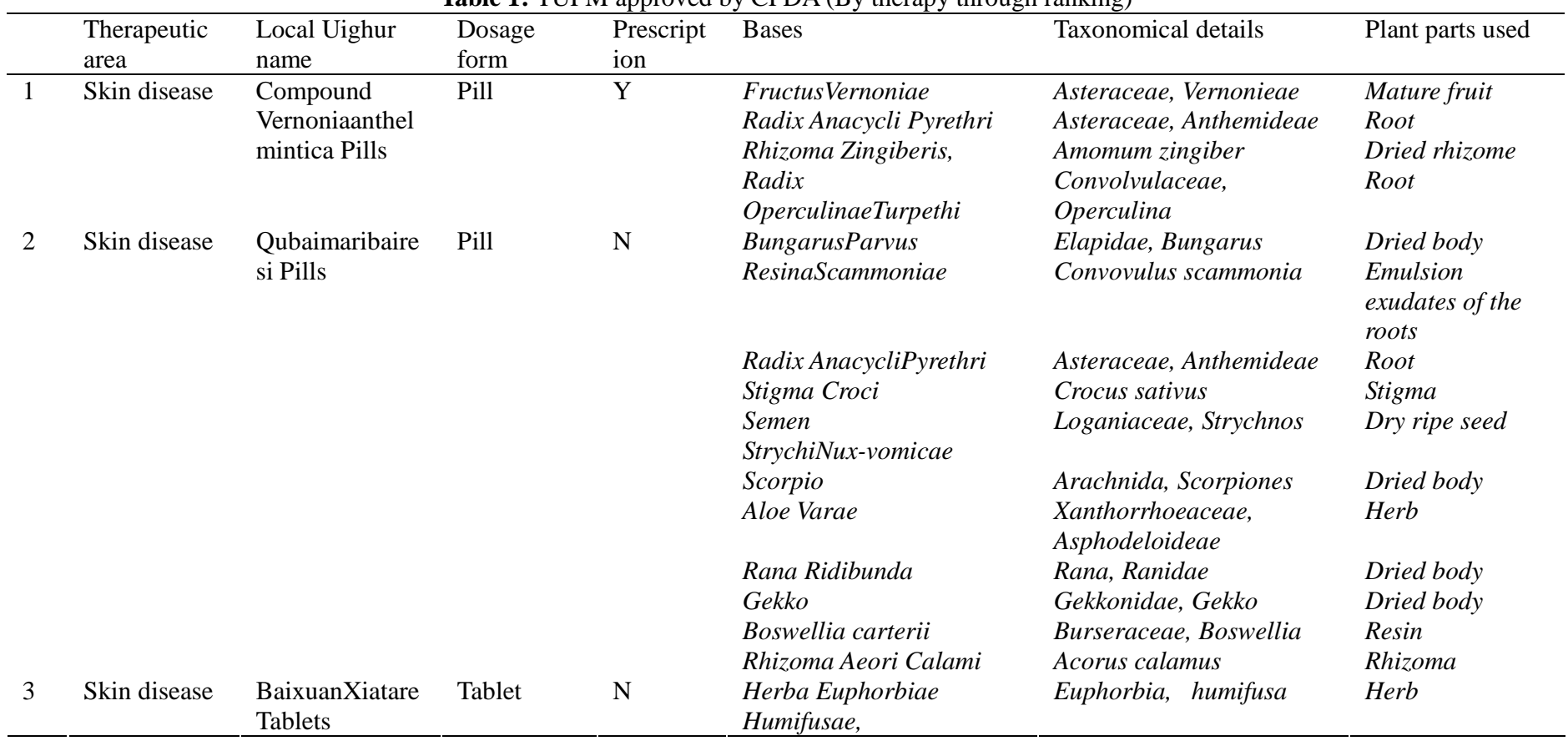


Fructus Chebulae,

Resina Scammoniae

Aloe Varae

$4 \quad$ Skin disease

QubaiBabuqi

Tablet

$\mathrm{Y}$

Tablets

Skin disease

Vernoniaanthel

mintica

Injection

$6 \quad$ Skin disease

Compound

Siyadan

Tincture

Skin disease

Kaliziran

Tincture

Fructus Psoraleae

Fructus Vernoniae

Rhizoma Alpiniae

Officinarum

Herba Operculinae

Turpethi

Fructus Vernoniae

SemenNigellae

Ganduliferae

Semen Persicae,

Fructus Granati

Fructus Vernoniae

Fructus Psoraleae
Skin disease

9
Compound

Siyadan Tonic

CompoundMun i Ziqi Granules
Tincture

Tincture

Y

Liniment

Granule
Injection $\quad \mathrm{Y}$

Radix et Herba

ovascular

11 Skin

disease, Cardi

ovascular

Disease
Yangxindawayi Soft

mixikemi

Unguent
Extract
Plumbaginis Zeylani-cae

Radix Polygni Multiflori

Radix Angelicae Sinensis

Radix Sapshnikoviae,

Fructus Cnidii

Cortex Dictamni

Fructus Mume

Semen sinapis

Flos Caryophylli

Semen Nigellae

Ganduliferae

Semen Persicae

FructusGranati

Herba cichorii

Cortex Foeniculi

Cichorium intybus $L$.

Fructus Apii

Herba Dracocephali

Semen Nigellae

Ganduliferae,

Radix Glycyrrhizae

Herba Chomomollae

Herba Cymbopogonis

Citrari

Fructus Ocimi Basilici

Fructus Pimpinellae Anisi

Herba Cymbopogonis

Distantis

Radix Arnebiae Euchroma

Herba Absinthii

Combretaceae, Terminalia Mature fruit

Convovulus scammonia

Emulsion

exudates of the

roots

Herb

Xanthorrhoeaceae

Asphodeloideae

Fabaceae, Psoralea

Asteraceae, Vernonieae

Zingiberaceae, Alpinia

Mature fruit

Mature fruit

Rhizoma

Convolvulaceae,

Herb

Operulina

Plumbaginaceae,

Plumbago

Asteraceae, Vernonieae

Root

Mature fruit

Ranunculaceae

Dry ripe seed

Rosaceae, Prunus

Dry ripe seed

Pomegranate, Granatum Mature fruit

Asteraceae, Vernonieae Mature fruit

Fabaceae, Psoralea

Knotweed, Fallopia

Apiaceae, angelica

Umbelliferae,

Saposhnikovia

Umbelliferae, Cnidium

Rutaceae

Rosaceae, Prunus

Cruciferae, Sinapis

Myrtaceae, caryophyllata

Ranunculaceae

Rosaceae, Prunus

Pomegranate, Granatum

Asteraceae, Cichorieae

Umbelliferae, Foeniculum

Asteraceae, Cichorium

Umbelliferae, Apium

Labiatae, Dracocephalum

Ranunculaceae

Mature fruit

Root tuber

Root

Root

Mature fruit

Peel

Near mature

fruits

Dry ripe seed

Flower bud

Dry ripe seed

Dry ripe seed

Mature fruit

Herb

Peel

Herb

Mature fruit

Herb

Dry ripe seed

Leguminosae, Glycyrrhiza

Malavaceae, Althaea

Root and rhizome

Herb

Herb

Ocimum, basilicum

Umbelliferae, Pimpinella

Poaceae, Cymbopogon

Mature fruit

Mature fruit

Herb

Boraginaceae, Arnebia

Root

Asteraceae, Artemisia

Hherb

Lignum Santali Albi

Santalaceae, Santalum

Moschus

Cervidae, Cervus

StigmaCroci.
ConcretioSiliceaBambusa
$e$

Iridaceae, Crocus

Gramineae, Bambusa
Lignum

Dry secretion in sachet of mature male Moschus

Heartwood

Cube after the

liquid secretion inside of haulm dried of plant 
Margarita,

Succinum

12 Genitourinar

y System

Luobufukebiri

Tablet

Tablets

Disease

13 Genitourinar

y System

Wenshensulafu

Disease

Mastiche

Resinatum

Preparata

Mastich
14 Genitourinar y System

Disease

Genitourinar

y System

Disease

Gujingmasiha Tablet N Tablets

KursiKaknaqyi

Tablet Y

y System

Tablet

Y
Aurum Foil,

Argentum Foil

Incuabulum Bombycis

Lignum Aquilariae

Pinus bungeana,

Fructus Dauci Sativae

Penis Bovis

Semen Amygdali Dulcis Semen Brassicae Rapae

Semen Cocois

Stigma Croci.

Arillus Myristicae

Mesua ferrea $L$.

Semen Medicaginis

Limoniumgmelinii (Wildl.)

Kuntze, etc.

Tuber Salep,

Semen Myristicae,

Rhizoma Alpiniae

Officinarum

Radix Aconiti Lateralis

Arillus Myristicae

Cortex Cinnamomi

Papaversom niferum $L$.

Stigma Croci.

Radix Anacycli Pyrethri

Fructus Cardamomi

Rhizoma Cyperi

Stigma Croci.

Semen Myristicae

Radix Curcumae Wenyujin

Flos Caryophylli

Radix et Rhizoma

Nardostachycis

Fructus Tsaoko

Flos Rosae Rugosae

Herba Physalis Alkekengi

Semen Cucumeris

Resina Draconis

Gum tragacanth

Ummi Rabicum

Semen Amygdali Dulcis

Radix Glycyrrhizae

Boswellia carterii

Frcutus Apii

Opium
Pteriidae

The resin of

Pinus plants, buried under the earth for long years and concreted and transformed to sulfhydryl

compound

Native gold

Native silver

Bombycidae

Burseraceae, Boswellia

Thymelaeaceae, Aquilaria

Pinaceae, Pinus

Apiaceae, Daucus

Bovidae

Rosaceae, Amygdalus

Cruciferae, Brassica

Alliaceae, Allicea

Crocus sativus

Myristicaceae, Myristica

Guttiferae, Mesua

Fabaceae, Medicago

Chenopodiaceae, lljinia

Orchidaceae, Bletilla

Myristicaceae, Myristica

Zingiberaceae, Alpinia

Cocoon shell of

silk cocoon

Aromatic resin of the mastic tree

Resinous wood

Not known

Mature fruit

Dry penis and

testicles of Bos

taurus

domesticus

Dry ripe seed

Dry ripe seed

Dry ripe seed

Stigma

Dry aril

Not known

Dry ripe seed

Not known

Tuber

Dry ripe seed

Rhizome

Ranunculaceae, Acontium

Secondary root

Myristicaceae, Myristica

Lauraceae, Cinnamamunz

Papaveraceae, papaver

Crocus sativus

Dry aril

Bark

Pericarpium

stigma

Root

Asteraceae, Pyrethrum
Burseraceae, Boswellia

Zingiberaceae, Amomum

Cyperaceae, Cyperus

Crocus sativus

Myristicaceae, Myristica

Zingiberaceae, Curcuma

Myrtaceae, Eugenia

Valerianaceae,

Nardostachys

Zingiberaceae, Amomum

Rosaceae, rosa

Spamaceae, Physalis

Cucurbitaceae, Cucumis

Palmae, Daemonorops

Leguminosae, Astragalus

Mimosaceae, Acacia

Rosaceae, Amygdalus

Leguminosae, Glycyrrhiza

Burseraceae, Boswellia

Umbelliferae, Apium

Papaveraceae, papaver

Orchidaceae, Bletilla

Secretions
Aromatic resin of

the mastic tree

Mature fruit

Rhizome

Stigma

Dry ripe seed

Root tuber

Flower bud

Root and rhizome

Mature fruit

Flower bud

Herb

Dry ripe seed

Processed resin

effused by the

fruit

Processed gum

Processed gum

Dry ripe seed

Root and rhizome

Resin

Mature fruit

Extract of fruit

Roots

Animal glands 


Disease
y System

Disease

18 Genitourinar y System Disease

Qiangshenluob ofusaiheli

Unguent
Soft

Extract
AmberaGrisea

Stigma Croci.

Semen

StrychiNux-vomicae

Boswelliacarterii

Penis Bovis

Semen Myristicae

FlosCaryophylli,

RhizomaAlpiniaeOfficinar

um

Papaversomniferum $L$.

Fructus Mori

Semen Euryales

RhizomaDioscoreaeSepte

mloba e

FructusRosaeLaevigatae

FructusGardeniae

Pinusbungeana

Pistaciavera L.

Semen Coryli,

Semen AmygdaliDulcis,

Papaversomniferum L.,

Herbalamiibarbati,

Semen Melo,

Semen Fraxini,

Cortex Cinnamomi,

Limoniumgmelinii (Wildl.)

Kuntze

Semen Cucumeris, Radix

CentaureaRuthenica, etc.

FlosNymphaeae

Radix cichorii

Fructus cichorii

FlosRosaeRugosae

Rhizoma et Radix

RheiPalmat

Folium FumicisDentati

Semen CuscutaeChinensis

Herbacichorii

Boswelliacarterii

Rhizoma Cyperi

Radix Aucklandiae

FructusQuerciAcutissimae

RhizomaZingiberis

Piper nigrum $L$.

22 Digestive

Tongzhiaitilefei Tablet

System

Disease lisana Tablets
$\mathrm{N}$

Folium Sennae

PhyllanthiFructus

FructusChebulaeImmatur us

FructusChebulae

FructusTerminaliaeChebu lae

$\begin{array}{ll}\text { Iridaceae, Crocus } & \begin{array}{l}\text { Digestive } \\ \text { Secretions }\end{array} \\ \text { Loganiaceae, Strychnos } & \text { Pistil } \\ & \text { Seeds } \\ & \text { Resin } \\ & \text { Bullwhip } \\ & \text { Fruit } \\ \text { Myristicaceae, Myristica } & \text { Buds } \\ \text { Myrtaceae, Syzygium } & \text { poppy capsule } \\ \text { Papaveraceae, Papaver } & \end{array}$

Zingiberaceae, Alpinia

Moraceae, Moraceae

Rhizome

Fruit

Nymphaeaceae, Euryale

Dioscoreaceae

Seeds

Tuber

Rosaceae, Rosa

Rubiaceae, Gardenia

Pinaceae, Pinaceae

Anacardiaceae, Pistacia

Betulac, Corylus

Rosaceae, Prunus

Papaveraceae, Papaver

Labiatae, Lamium

Cucurbitaceae, Cucumis

Oleaceae, Ligustrum

Lauraceae, Cinnamomum

Plumbaginaceae, Limoni um

Cucurbitaceae

Asteraceae, Centaurea

Nymphaeaceae,

Nymphaea

Asteracea, Cichorium

Asteracea, Cichorium

Rosaceae, Rosaceae

Apiaceae, Ligusticum

Polygonacea, Rheum

Boraginaceae, Anchusa

Convolvulaceae, Cuscuta

Asteraceae, Cichorium L.

Fruit \& seeds

Fruit

pine cone

Fruit

Fruit

Fruit

Seeds

Herb

Seeds

Fruit

limb

Herb

Seeds

Root

Flower

Roots

Fruit

flower

Herb

Root

Herb

Herb

Herb

Burseraceae, Boswellia

resin

Cyperaceae, Cyperus

Rosaceae

Stem

Stem

Seeds

Roots

Zingiberaceae, Zingiber

Piperaceae, Piper

Seeds

Leguminosae, senna

foliage

Combretaceae, Terminalia

Fruit

Phyllanthace, Phyllanthus

Fruit

Combretacea, myrobalan Fruit

Combretaceae, Terminalia Fruit 
Disease

24 Digestive

System

Disease

25 Digestive

System

Disease
Digestive

System

Disease

27 Digestive

System

Disease
Disease,mus culoskeletal system

(rheumatis

m)

System

Disease

Respiratory

System

Disease
Rose Oral

Liquid

Mamuranantidi

arrheal

Capsules
Granules

Mixture

$\mathrm{N}$

Capsule

Y

HuganBuzure

Granules

Sanhanyao Tea

Liniment

N

Soft

Extract

Granule

$\mathrm{N}$

N

Y

Y

FructusApii,

Herbacichorii

Semen Cuscutae

Chinensis.

Radix Api

Cortex Foeniculi

Cichoriumintybus L.

FructusFoeniculi

Fructus Cardamomi

Valerianaofficinalis Linn.

FructusPimpinellaeAnisi

Cortex Cinnamomi

FructusTsaoko

FructusCinnamomi

RhizomaAlpiniaeOfficinar

um

FructusPiperisLongi

FlosCaryophylli

FructusFoeniculi

FructusApii

FructusGardeniae

FructusCordiaeDicho

tomae,

FructusJujubae

Papaversomniferum $L$

Radix Glycyrrhizae

FructusAlthaeaeRoseae

Semen Cucumeris

Semen Cydoniae

Semen AmygdaliDulcis

Papaversomniferum $L$.

Radix Glycyrrhizae

UmmiRabicum

RhizomaKaempferiae

FlosNymphaeae

FructusCordiaeDichotom

ae
Rosaceae, Rosaceae

Flower

anunculacea, Coptideae

palmae

Burseraceae, Boswellia

Rootstock

Resin

Resin

I

Lythraceae, Punica

Larva

Pericarp

Fungus

Herb

Plantaginaceae, Plantago

Phyllanthaceae,

Fruit

Phyllanthus

Apiaceae, corainder Herb

Combretacea, Quisqualis Fruit

Berberidaceae, Berbens

Rosaceae, Rosaceae

Rootstock

Flower

Umbelliferae, celery Seeds

Asteraceae, Cichorium L. Herb

Convolvulaceae, Cuscuta Herb

europaea

umbelliferae, celery Roots

Umbelliferae, Foeniculum Roots Mill.

I

Asteraceae, Cichorium L. Roots

Zingiberaceae, Elettaria Seeds

Maton

Valerianaceae,Valeriana Herb

Apiaceae, Pimpinella Seeds

Lauraceae, Cinnamomum Rind

Zingiberaceae, Amomum Fruit

$L$.

Lauraceae, Cinnamomum Fruit

Zingiberaceae, Alpinia Roots

Piperaceae, Piperaceae Cluster

Oleaceae, Oleaceae Flower

umbelliferae, Foeniculum Seeds

Mill

umbelliferae, celery Seeds

Rubiaceae, Gardenia Fruit

Boraginaceae, Cordia Fruit

Rhamnaceae, Ziziphus $\quad$ Fruit

Papaveraceae, Papaver Pericarpium papaveris

Root

Seeds

Seeds

Cucurbitaceae, Cucumis

Linn

Rosaceae, Cydonia

Rosaceae, Prunus

Papaveraceae, Papaver

Fabaceae, Glycyrrhiza

Seeds

Seeds

Seeds

Roots

Leguminosae, Acacia Gum

Zingiberaceae, Stem

Kaempferia

Nymphaeaceae,

Flower

Nymphaea

Boraginaceae, Cordia Fruit 
System

Disease

32 Blood System

33 Blood System
Granules

Kebireti Tablets Tablet

$\mathrm{Y}$

GangkangMuku Tablet

li Tablets
Hanchuanzupa

FructusJujubae
HerbaChomomollae
Radix Glycyrrhizae
Semen AlthaeaeRoseae
Rhizoma et Radix
RheiPalmat
Papaversomniferum L.

HerbaHyssopi

Herbaadianticaudati

Radix Glycyrrhizae

FructusFoeniculi

FructusApii

Semen Trigone

HerbaCymbopogonisDista ntis

FlosRosaeRugosae

Semen Urticae

Sulfur

UmmiRabicum,

Sal-Ammoniac

Radix AnacycliPyrethri

Poria

Com-miphoramukul

Succinum

OsCorallii

Concha

MargaritiferaUsta

FructusChebulae

FructusTerminaliaeChebu

lae,

PhyllanthiFructus

FructusChebulaeImmatur

us

RhizomaPolygoniBistorta

e

IncuabulumBombycis

HerbaAnchusae,

Radix et

RhizomaNardostachycis

Flos Salix Caprea

Moschus

Stigma Croci.

HerbaDracocephaliMolda

vicae

HerbaLavandulae

FlosAnchusae

Usnea

FlosRosaeRugosae, etc.

HerbaDracocephaliMolda vicae,
Rhamnaceae, Ziziphus uya Granules

Granule
Tongzhisurunjia Capsule Y ng Capsules
FructusCardamomi

Compositae, Anthemis

Fabaceae, Glycyrrhiza

Malvaceae, Alcea

Polygonaceae, Rheum

Papaveraceae, Papaver

Labiatae, Hyssopus

Adiantaceae, Adiantum

Fabaceae, Glycyrrhiza

Apiaceae, Foeniculum

Apiaceae, Apium

Fabaceae, Trigonella

Rutaceae, Ruta

Rosaceae, Rosa

Urticaceae, Urtica L

Sulphur

Leguminosae, Acacia

Sal-Ammoniac

Asteraceae,

Chrysanthemum

Fomitopsidaceae, Wolfipor

ia

Burseraceae

Ambrum

Agariciidae

Nacre

Combretaceae, Terminalia

Combretaceae, Terminalia

Combretaceae, Terminalia

Phyllanthaceae,

Phyllanthus

Polygonaceae, Polygonum

Silkworm cocoon

Boraginaceae, Lycopsis,

Echium

Caprifoliaceae,

Nardostachys

Salicaceae, Salix

Moschus

Iridaceae, Crocus

Labiatae, Dracocephalum

Lamiaceae, Perilla L.

Boraginaceae, Lycopsis,

Echium

Zingiberaceae, Elettaria

Maton

Usneaceae, Usnea

Rosaceae, Rosa

Labiatae, Dracocephalum
$D(+)$-Sucrose

Dextrin

Colchicaceae, Colchicum

Convovulus scammonial

Iridaceae, Crocus
Fruit

Flower

Roots

Seeds

Roots

Pericarpium papaveris

Lea

Hibiscus trionum

Root

Fruit

Fruit

Seeds

Lea

Flower

Seeds

Crystal

Gum

Crystal

Roots

Sclerotium

Resin

Resin

Calcareous

skeleton

Powder

Fruit

Fruit

Fruit

Fruit

Stem

Silkworm cocoon Lea

Roots and rhizomes

Flower

Sachet

Stigma

Hibiscus trionum

Seeds

Flower

Seeds

Thallus

Flower

Hibiscus

trionumapp:add word:Hibiscus

trionum

$D(+)$-Sucrose

Dextrin

Capsule

ResinaScammoniae

Stigma Croci. 


$\begin{array}{ll}\text { Musculoskel } & \text { Compound } \\ \text { etal system } & \text { Luotuopengzi } \\ \text { (rheumatism) } & \text { Ointments }\end{array}$

39

$\begin{array}{ll}\text { Nervous } & \text { Compound } \\ \text { system } & \text { Gaoziban } \\ & \text { Tablets }\end{array}$

40

Nervous system

JianxinHemi'er gaozibanAnbire Tablets

41 Nervous system

Xingnaokukeya Tablet Tablets

ChuzhangZehai Tablet pu Tablets

system,ophth almology

Tablet

Ointment

N

$\mathrm{N}$

$\mathrm{N}$

43 Anti-infectio A'naerFujie Ye Lotion us agent

Folium Sennae
FructusChebulae
HerbaOperculinaeTurpeth
i
Semen AmygdaliDulcis
Semen Pegani,
Semen HyoscyamiNigeris

HerbaAnchusae
Radix
CentaureaRuthenica,
Lignum Santali Albi
Limoniumgmelinii(Wildl.)
Kuntze
HerbaDracocephali

Semen LepidiiSativi FructusPerillaeArgutae FlosAnchusae

IncuabulumBombycis

HerbaLavandulae

FructusCoriandri.

HerbaAnchusae

FlosAnchusae,

AmberaGrisea
Margarita
Succinum
IncuabulumBombycis
Os Corallii

LignumSantali Albi

AurumFoil

ArgentumFoil

FlosSalixCaprea, etc.

Aloe Varae

HerbaAbsinthii

Mastiche

ResinaScammoniae

FructusColocynthis

ResinaScammoniae,

Aloe Varae.

Stigma Croci.

HerbaOperculinaeTurpeth

$i$

FructusChebulae

FlosRosaeRugosae

Boswelliacarterii

CortexGranatii,

FrcutusSophorii

FructusCnidii

GallaTurcica,

OsCorallii

FructusZanthoxyli

Borneo
Leguminosae, senna

Combretaceae, Terminalia

Convolvulaceae,

Operculina

Rosaceae, Prunus

Peganaceae, Peganum

Solanaceae, Hyoscyamus

Lea

Fruit

Velamen

Seeds

Seeds

Seeds

Boraginaceae,

Lycopsis,Echium

Asteraceae, Centaurea

Santalaceae, Santalum

Plumbaginaceae,

Limonium

Labiatae, Dracocephalum

Brassicaceae, Lepidium

Lamiaceae, Perilla L.

Boraginaceae,

Lycopsis, Echium

Silkworm cocoon

Lamiaceae, Lavandula

Apiaceae, Coriandrum

Boraginaceae, Lycopsis,

Echium

Boraginaceae, Lycopsis,

Echium

Ambergris

Pernulo

Ambrum

Silkworm cocoon

Agariciidae

Santalaceae, Santalum

Native gold

Native silver

Salicaceae, saliko

Liliaceae, Aloe

Compositae, artemisia

Burseraceae, Boswellia

Convovulus scammonial

Cucurbitaceae

Convovulus scammonial

Liliaceae, Aloe

Iridaceae, Crocus

Convolvulaceae,

Operculina

Combretaceae, Terminalia

Rosaceae, Rosa

Burseraceae, Boswellia

Punicaceae, punica

Leguminosae, Sophora

Umbelliferae, Cnidium

Gall wasps

Agariciidae

Rutaceae, Zanthoxylum

Dipterocarpaceae,

Dryobalanops
Lea

Roots

Roots

Lea

Hibiscus

trionumapp:add

word:Hibiscus

trionum

Seeds

Seeds

Flower

Silkworm cocoon

Flower

Seeds

Lea

Flower

Bezoar

Pernulo

Resin

Silkworm cocoon

Calcareous

skeleton

Bole

Native gold

Native silver

Flower

Lea

Lea

Resin

Gum

Fruit

Gum

Lea

Stigma

Velamen

Fruit

Flower

Resin

Pericarp

Root

Fruit

Larva

Calcareous

skeleton

Seeds

Resin

\section{Analysis of TUPM patents}

Among all the registered TUPMs, 14 TUPMs are related to totally 19 invention patent applications that were mostly submitted after 2000 . Consequently 10 TUPMs got patent authorization, mainly focusing on the treatment of skin disease, urogenital disease and respiratory disease. In detail, 5 TUPMs are for skin disease, 2 for urogenital disease and one for each aspect of respiratory, digestive and ophthalmology disease.

Most of these TUPM patents are related to prescription composition. Only the patent of Xi-payimaibizi Liquid involves with quality control 
http://dx.doi.org/10.4314/ajtcam.v11i2.13

and detection and is named "A quality control method to treat prostatitis using traditional Chinese medicine". The patents of Kaliziran Tincture and Vernoniaanthelmintica Injection are new application patents, named as "Anew use of Kaliziran Tincture to treat or prevent psoriasis" and "A major use of Vernoniaanthelmintica Injection in the data basis of cough-asthma" respectively. Moreover, only Zukamu Granules has got new drug dosage form patent, named as "A preparation and method of cold capsule". (see Table 2)

Table 2: TUPM with patent authorization

\begin{tabular}{|c|c|c|c|c|c|}
\hline No. & $\begin{array}{l}\text { Therapeutic } \\
\text { areas }\end{array}$ & Drug names & Patent names & Patent content type & $\begin{array}{l}\text { Applicatio } \\
\text { n time }\end{array}$ \\
\hline 2 & $\begin{array}{l}\text { Skin disease, } \\
\text { cardiovascular } \\
\text { disease }\end{array}$ & Suziafu Tablets & $\begin{array}{l}\text { A preparation method of traditional } \\
\text { Chinese medicine composition }\end{array}$ & Prescription & 2010 \\
\hline 3 & Skin disease & BaixuanXiatare Tablets & $\begin{array}{l}\text { A TCM preparation and method to treat } \\
\text { skin disease }\end{array}$ & Prescription & 2008 \\
\hline 4 & Skin disease & Kaliziran Tincture & $\begin{array}{l}\text { A new use of Kaliziran Tincture to treat or } \\
\text { prevent psoriasis }\end{array}$ & New Application & 2006 \\
\hline 5 & Skin disease & $\begin{array}{l}\text { Vernoniaanthelmintica } \\
\text { Injection }\end{array}$ & $\begin{array}{l}\text { A major use of Vernoniaanthelmintica } \\
\text { Injection in the catabasis of cough-asthma }\end{array}$ & New Application & 2004 \\
\hline 6 & $\begin{array}{l}\text { Urogenital } \\
\text { disease }\end{array}$ & Xi-payimaibizi Liquid & $\begin{array}{l}\text { A quality control method to treat prostatitis } \\
\text { using traditional Chinese medicine }\end{array}$ & Quality Control & 2010 \\
\hline 8 & $\begin{array}{l}\text { Digestive } \\
\text { disease }\end{array}$ & HuganBuzure Granules & $\begin{array}{l}\text { An extraction technology of compound } \\
\text { HerbaCichorii }\end{array}$ & Prescription & 2009 \\
\hline 9 & $\begin{array}{l}\text { Respiratory } \\
\text { disease }\end{array}$ & Zukamu Granules & A preparation and method of cold capsule & New dosage form & 2002 \\
\hline 10 & $\begin{array}{l}\text { Ophthalmology } \\
\text { disease }\end{array}$ & XuzhangZehaipu Tablets & $\begin{array}{l}\text { A preparation method of traditional } \\
\text { Chinese medicine to treat cataract }\end{array}$ & Prescription & 2008 \\
\hline
\end{tabular}

\section{Analysis of TUPM publication}

Data analysis of TUPM publications in CNKI showed a steady growth since 2001 to 2011 (see Figure 2). Especially after 2007 there is a rapid increase of paper quantity, implying greater research interest in TUPM.

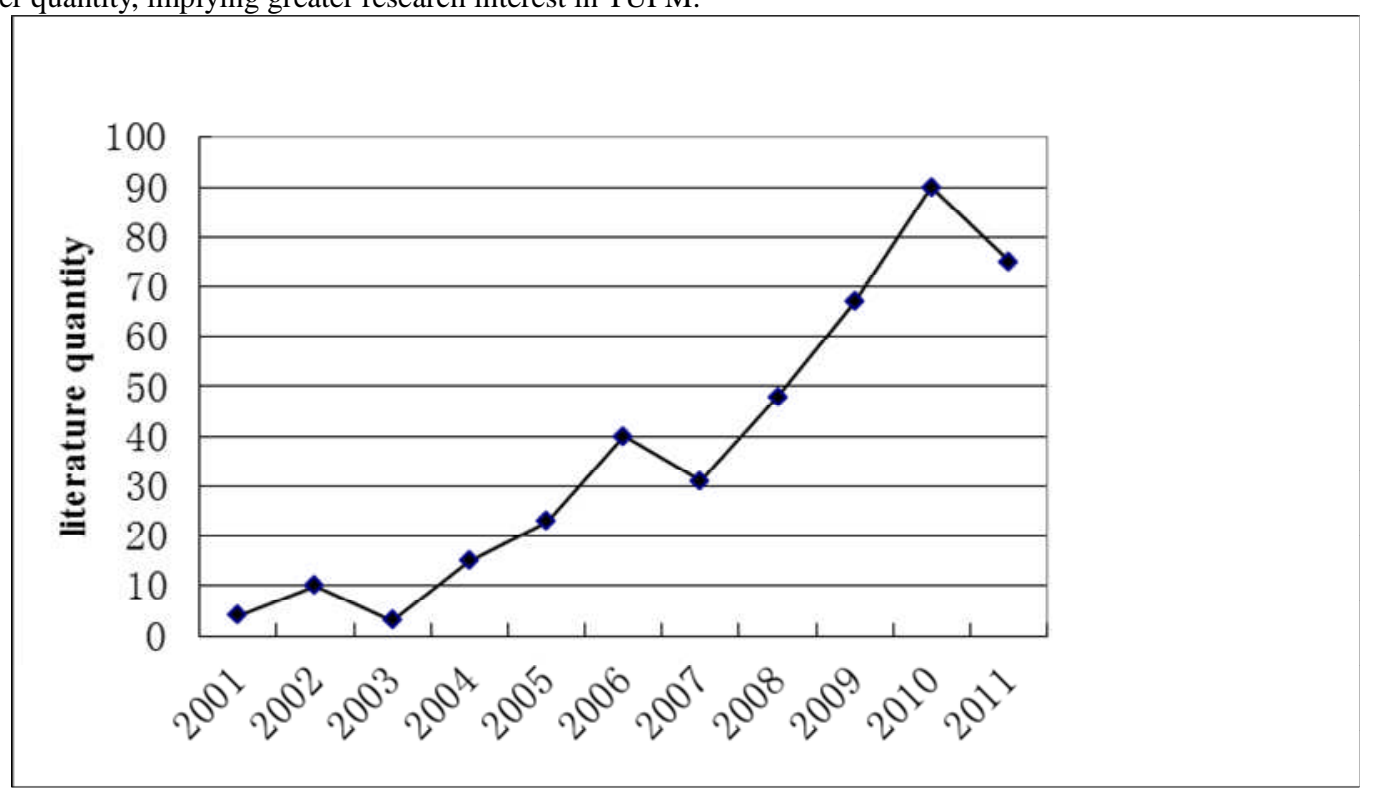

Figure 2: Publications of TUPM in CNKI (2001-2011)

Through sorting the number of academic publications, the first ten TUPMCs were summarized in Table 3. Medicines for treating skin disease are still the largest group in number, including Kaliziran Tincture, BaixianXiatare Tablets, Compound MuliZiqi Granules, 
http://dx.doi.org/10.4314/ajtcam.v11i2.13

Vernoniaanthelmintica Injection. The other TUPMs are in the therapeutic areas of urogenital, respiratory, cardiovascular, anti-infection disease and rheumatism. Among all the 408 research articles of TUPM, the maximum quantity appears on the dentistry medicine Xipayi mouth rinse, with 94 academic publications. Xipayimouth rinse is used for sterilization, anti-inflammation, protecting the tooth and oral soft tissues. It's a single-formula preparation with Quercus infectoria Olivas the effective components. It is widely used in Southern Xinjiang Province of China not only by Uighur medicine doctors for treating Abnormal Savda Syndrome but also by ordinary people for self-medication.

In the respect of research contents, there are 258 clinical observation publications, $63.2 \%$ of the total. The rest publications are related to basic researches, quality standard and preparation technology, accounting for $10.8 \%, 9.3 \%$ and $2.0 \%$ respectively. In detail, publications about Zukamu Granules and A'naerFujie Ye are highly related to quality standard, and the number of these publications about quality standard accounts for about $30 \%$ and $50 \%$ respectively. But there is no clinical observation research in the publications of A'naerFujie Ye. In the publications of Aiweixin Liquid, basic research and clinical observation research have nearly the same quantity, each about $34 \%$ of the total. (See Table 3)

Table 3: Academic publications of ten mostly studied TUPM

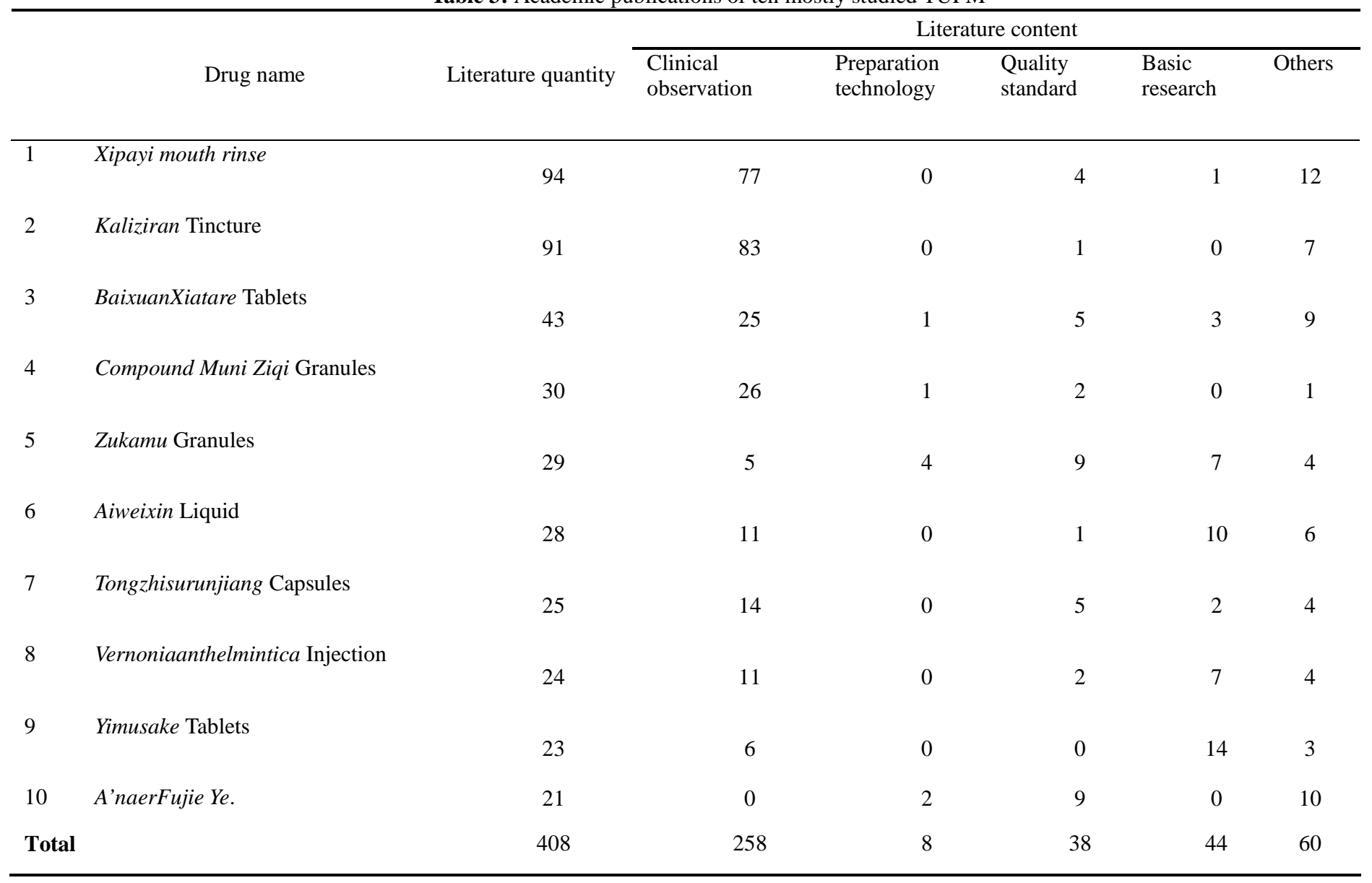

\section{Analysis of Uighur herb patents}

Sorted by the usage frequency in the 43 approved TUPMs, the ten mostly used Uighur herbs can be ordered from high to low as: ResinaScammoniae, Folium FumicisDentati, HerbaDracocephali, Semen AmygdaliDulcis., HerbaChamomillae, FructusPimpinellaeanisi, Cortex Foeniculi, FructusVernoniae, FructusApii, and Radix AnacycliPyrethri.

For patent applications of these ten main Uighur herbs, there are 274 patent applications totally. Among these patent applications, 24 applications have got patent authorizations, with an average authorization rate of $12.4 \%$. The main therapeutic areas of these Uighur herbs include rheumatism, skin disease, urogenital disease and cosmetics (see table 4). Herba Chamomillae has the most patent authorizations, with 13 authorizations from 120 patent applications. Most of its patents focus on the cosmetics area, which are widely used as natural skin care extractions in making hand cream, cleanser and bath cream.

The following Uighur herb is FructusVernoniae, with 20 applications and 5 authorizations. These patents are mainly related to the treatment of vitiligo and other skin diseases, except one patent about cough-asthma, which was named as "A major use of Vernonia anthelmintica Injection in remission stage of cough-asthma”.

The mass of patent applications of Folium FumicisDentati concentrate on the extraction technology and preparation process, and half of these patent applications are used to treat tumor and cardiovascular disease. There are two authorized patents involved in two medicines, which were named as "Gandanweifukang Mixture (a medicine for liver and gastrointestinal disease) and its preparation method" and "Abnormal SavdaMunziq and its preparation method" respectively. The patent applications of Semen AmygdaliDulcis are mainly related to the therapeutic areas of rheumatism and urogenital disease, and two authorized patents are "Abnormal SavdaMunziq and its preparation method" and "Improved traditional patent medicine to relieve pain and act as a diuretic". Furthermore, seven patent applications of FructusApiiare mainly used in the treatment of hypertension, hyperglycemia, and rheumatic diseases, and all of them are about TUM's prescriptions.

The other Uighur herbs' patent applications got no patent authorizations. Herba dracocephali has 16 patent applications, mainly focusing on the research of high flavonoids content, which indicates significant treatment effect towards cardiovascular disease. These patent applications involve some preparations such as sustained release tablets and orally disintegrating tablets of flavones in Herba dracocephali. In addition, 
http://dx.doi.org/10.4314/ajtcam.v11i2.13

FructusPimpinellaeanisi has no patent applications until now.

Table 4: Patent analysis of main Uighur herbs

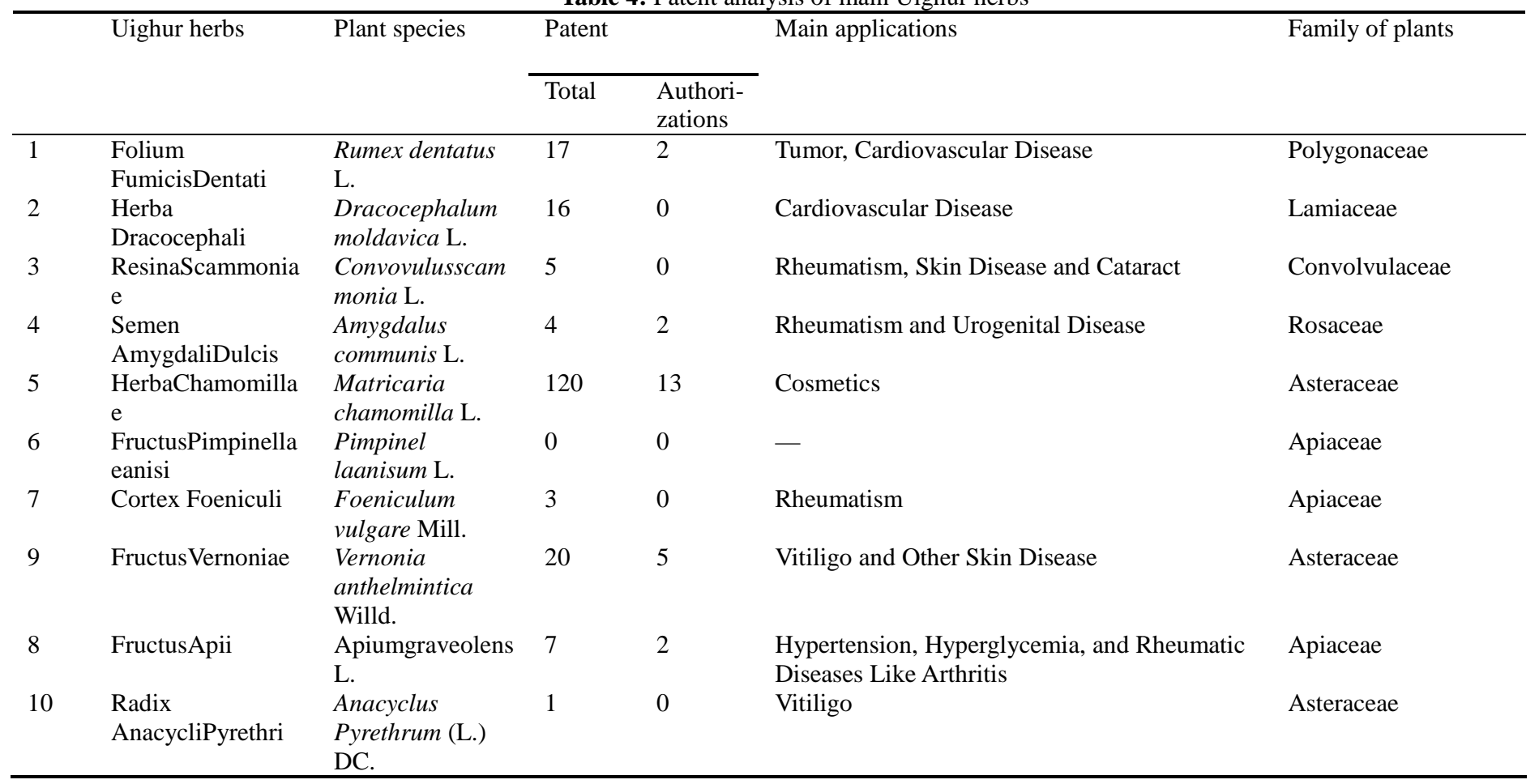

\section{Analysis of Uighur herb publications}

The ten frequently used Uighur herbs have 455 academic publications totally. Among these publications, only $50 \%$ are directly related to medicine research. These literatures mostly concentrate on the extraction of the chemical composition and pharmacological activity analysis, accounting about $26.4 \%$ of the total publications, followed by clinical effect researches (as shown in Table 5). Meanwhile, the topic concentrations of research literatures are consistent with the patent applications of the Uighur herbs.

HerbaDracocephali has the largest amount of research publications, about 139. These researches mainly concentrate on the study of chemical component, including methods of flavonoids determination, HPLC fingerprint and pharmacologic actions of asthmatic rats. In the aspects of clinical effects, studies mainly focus on the influence of coronary heart disease and patients' curative effect observation (Gu et al., 2004; Ren et al., 2011; Song et al., 2010; Tian et al., 2012).

The number of research articles for HerbaChamomillae is about 138, most of which are reports about some cosmetics products. FructusVernoniae has 106 research publications, most of which are medical related, including about 43 chemical component studies and 44 clinical researches.

Especially, clinical effects researches concentrate on the clinical curative effect observation of Vernoniaanthelmintica Injection. Academic publications of Folium FumicisDentatiare are mainly about cultivation, planting and medicinal clinical effects researches. Comparatively, the publications of ResinaScammoniae are less, which are mostly related to TUM preparations, including the quality standard of BaixuanXiatare Tablets and Kukeya Tablets, and the clinical effects of Tongzhisurunjiang Capsules and BaixuanXiatare Tablets. Meanwhile, researches about Semen AmygdaliDulcis are also found to be few: for preparations, there are two research articles including Tongzhisurunjiang Tablets and Tongzhisurunjiang Capsules (Yao, 2012); there are 8 articles about chemical component analysis; and 4 papers about cultivation and planting. Among the 12 research articles of FructusApii, two are involved with the preparations of HanchuanZupa Granules and Ganbaokang Granules.

Radix AnacycliPyrethri has only one research article as well as Cortex Foeniculi, titled as "The quality standard research of TUM preparation Gujingmaisiha Tablets" and "The study of chemical component of Cortex Foeniculi" respectively. In addition, FructusPimpinellaeanisi has no academic publication.

Table 5: Publication analysis of main Uighur herbs

\begin{tabular}{|c|c|c|c|c|c|c|}
\hline \multirow{2}{*}{ Herb } & \multirow{2}{*}{ Literature quantity } & \multicolumn{5}{|c|}{ Literature content } \\
\hline & & Preparations & Clinical effects & Component and activity & Cultivation and planting & Others \\
\hline Resina Scammoniae & 5 & 4 & 1 & 0 & 0 & 0 \\
\hline Folium Fumicis Dentati & 33 & 0 & 3 & 1 & 3 & 26 \\
\hline HerbaDracocephali & 139 & 10 & 21 & 65 & 10 & 33 \\
\hline Semen AmygdaliDulcis. & 8 & 2 & 1 & 1 & 0 & 4 \\
\hline HerbaChamomillae & 138 & 1 & 1 & 8 & 4 & 124 \\
\hline FructusApii & 12 & 2 & 0 & 1 & 0 & 9 \\
\hline Radix AnacycliPyrethri & 1 & 1 & 0 & 0 & 0 & 0 \\
\hline FructusVernoniae & 106 & 7 & 44 & 43 & 1 & 11 \\
\hline $\begin{array}{l}\text { FructusPimpinellaeanis } \\
i\end{array}$ & 0 & 0 & 0 & 0 & 0 & 0 \\
\hline Cortex Foeniculi & 1 & 0 & 0 & 1 & 0 & 0 \\
\hline
\end{tabular}


Table 6: Contents of publications

\begin{tabular}{|c|c|c|c|}
\hline Herb & $\begin{array}{l}\text { Component and activity } \\
\text { literature quantity }\end{array}$ & Phytochemical constituents & Published pharmacological activity reports \\
\hline Folium Fumicis Dentati & 1 & $\begin{array}{l}\text { Chrysophanol, Emodin, } \\
\text { Aloe-emodin, Physcion, } \\
\text { Phytosterol, Phytosterol }\end{array}$ & $\begin{array}{l}\text { Research on the active ingredients of hemostasis } \\
(\mathrm{Xu}, 1981)\end{array}$ \\
\hline
\end{tabular}

Herba Dracocephali 65

Sorbarin, Scutellarein
Rhamnoside, Linarin

Semen Amygdali Dulcis. $\quad 1$

HerbaChamomillae

8

Fructus Apii

1

Radix Anacycli Pyrethri

0

Fructus Vernoniae

43

Fructus Pimpinell aeanisi 0

Cortex Foeniculi 1
Anisole

Vegetable oil, Protei, Starch、Vitamin A、B1、 B2,Digestive Enzyme ChaPaxulene, Proazulere, Farnesene, Bisabolol, A-bisabolol Oxide-A, Suaianolide, Matrioin, Matricarin Eucalyptol, Umbrella Lactone, Celery Element, Linolenic Acid, Volatile Oil, Flavonoids, Boron, Minerals

Volatile Oil: Caryophyllene, $\beta$-pinene, Ethybutylether Volatile Oil

Fennel essential oil, Polysaccharide, Anethole
Review research of chemical component and pharmacological activity (Song et al., 2010); research on the chemical component of Herba Dracocephali (Gu et al., 2004); chemical constituents and their pharmacological activities research (Yang et al., 2013), etc. No

Radiation treatment of herb tea for the reduction of microbial contamination (Flores chamomillae) (Katušin-Ražem et al.,1983), etc.

Thin layer chromatography for characterization of pharmacopeia drugs (Stahl,1969), etc. No

No

Explore the effect of the Vernoniaanthelmintica wild Injection on the lymphocyte subclass of mice (Deng et al., 2002). The content of flavonoids in the fruit and various medical forms of fructus vernonia is tested by spectrophotometry (Zhou et al., 2000), etc. No

Investigate the in vitro anti-candidal activity of the essential oil of Illicium verum (EOIV) alone and in combination with fluconazole. (Zhao et al., 2004), etc.

\section{Discussion}

Through the analysis of TUM registry, patent and academic publications, this study found that that most of research and development of TUM focus on the therapeutic areas of skin disease, urogenital disease, rheumatism and digestive system disease, implying the medical advantages of TUM in these therapeutic areas.

Publications about the popular Uighur herbs can also validate the effectiveness of those plant species used in the above main therapeutic areas. It is worth noting that researches about the active ingredients of Uighur herbs have been either old or rare (Xu, 1981; Katušin-Ražem et al., 1983; Stahl,1969). And the researches in recent years focus on the pharmacological activities and the actual clinical application test. Some of plant species are highly considered, such as Herba Dracocephali (Song et al., 2010; Gu et al., 2004; Yang et al., 2013), Fructus Vernoniae (Deng et al., 2002). Those plants have been used for a very long term and still can be used in today with scientific method validated.

Among all the research work about TUM, researches about skin disease account for the largest part, mainly focusing on the treatment of vitiligo and psoriasis. It may have a high relevance to the high morbidity of these two skin diseases in Xinjiang (Wen et al., 2013). Because these skin diseases are significantly influenced by climate, Xinjiang with intense sunlight and arid climate has higher skin disease morbidity (Tang et al., 1998). BaixuanXiatare Tablets, Kaliziran Tincture and Vernoniaanthelmintica Injection are famous medicines used for skin diseases such asvitiligo and psoriasis in Xinjiang, and these medicines are also widely used in Uighur hospitals (Liang, 2011; Liu and Liang, 2012; Wang, 2012; Wei et al., 2009). At present, more attentions are paid on the researches of Kaliziran Tincture and Vernoniaanthelmintica Injection, whose major components are from Uighur herb ofVernoniaAnthelmintica L.Vernoniaanthelmintica Injection is a single-formula preparation, also the only injection of TUPM. In particular, some pharmacological researches proved the treatment mechanism of VernoniaAnthelmintica L, showing that VernoniaAnthelmintica $L$ could enhance the activity of tyrosinse and melanin synthesis in A375 cells and increase the tyrosinase mRNA expression, increase melanin synthesis from gene level (Deng et al., 2004; Hui et al., 2010; Ma et al., 2008).

Although there are quite a few species of TUM related to urogenital disease, few researches and patent authorizations are carried out until now. The main Uighur patent medicines related to urogenital disease are Xi-payimazibizi Liquid treating prostatitis and KursiKaknaqused for urinary tract infection. They also have significant component differentia. For the reason of less research articles about the two TPUM, except for a few clinical observation researches, the knowledge about their function mechanism and the effective chemical composition is rare.

Rheumatism belongs to musculoskeletal disease in the ATC classification system. Tongzhisurunjiang Capsules is the most famous anti-rheumatism medicine in Xinjiang. Evidences form pharmacological and clinical study show that Tongzhisurunjiang Capsules can relieve the 
http://dx.doi.org/10.4314/ajtcam.v11i2.13

symptom of acute gouty arthritis patients effectively and promptly (Fan et al., 2009; Zhu et al., 2013), reduce the ESR and c-reactive protein, and also suppress expression of IL-1 $\beta$, TNF- $\alpha$ in serum. It's proved to be an effective way to treat the acute gouty arthritis (Yao, 2012).

At last, this study showed there are several TUPMs for treating digestive system disease. However, only HuganBuzure Granules got patent authorization. This medicine is aiming at the treatment of stomachache, cirrhosis, hepatitis and other diseases, with few research articles and clinical efficacy evidence. Additionally, extractions of HuganBuzure Granules have protective effect on liver injury in mice (Wu et al., 2011).

HerbaDracocephali, FructusVernoniae, HerbaChamomillae and FoliumFumicisDentati have been mostly studied Uighur herbs. Most of literatures on HerbaDracocephali focus on the content of flavonoids compounds and pharmacological activity (Yan et al., 2003). The studies show that the flavones of HerbaDracocephali could inhibit the proliferation of VSMC induced by TNF- $\alpha$, which might be one of the pharmacological mechanisms of HerbaDracocephali total flavoes in treatment for atherosclerosis (Cao et al., 2011; Song et al., 2010). In clinical curative effect, there is less study on HerbaDracocephali. FructusVernoniae is the major components of pharmaceutical preparation in treating psoriasis and vitiligo, and major research is conducted on chemical composition and extraction technology (Yao, 2007; Yu et al., 2007). HerbaChamomillaehas protective effect to skin and it is widely used in cosmetics for research has shown the anti-inflammatory effect of volatile oil from it (Yuan et al., 2011). The study of Folium FumicisDentati is mainly on the extraction process of flavonoids. However there is no research on pharmacological action and clinical effect (Nurma-mmatet et al., 2008; Palida et al., 2013).

In general, while the curative effect of TUM has been proved to some degree, lacks of using modern technologies may cause uncertain effect, which has affected the standardization of TUM. The shortage of applying modern technologies has limited TUM in drug-approval and medical application.

\section{Conclusion}

As a part of China and Central Asia's traditional medicine, TUM in Xinjiang play an important role in the local care system, at the same time, it promotes the cross-regional communication and development of health. This study finds that the TUM in Xinjiang has a vital value and abundant experience in treatment of skin disease, urogenital system disease, rheumatism and respiratory system disease. On the one hand, this study can provide some new ideas and methods for these diseases' treatment, especially for patients with some intractable diseases such as psoriasis and vitiligo. On the other hand, traditional Uighur herbs can be a reservoir of traditional medicinal plants with potential for the development of some diseases' modern therapeutics. These traditional Uighur herbs with excellent curative effect should be screened in detail for their phytochemical properties and pharmacological activity to discover new bioactive constituents, then provide knowledge base for new drug's R\&D. Further, more studies for TUM should be carried out, and the efficacy and safety of TUM should be evaluated for pharmacological studies especially the species with high value.

\section{Acknowledgements}

This study is supported by the research funding of University of Macau MYRG160(Y2-L2)-ICMS11-HH).

\section{References,}

1. Amat, N., Upur, H., Ablimit, A. and Matsidik, A. (2009). Immunomodulatory effects of Abnormal Savda Munsiq, a traditional Uighur medicine, on the combined stress mice. Journal of Ethnopharmacology, 122: 42-47.

2. Cao, W.J., Xing J.G., Wang X.C., and Jin J.G. (2011). Inhibitory Effects of Dracocephalum Total Flavoes on Proliferation of Rat Vascular Smooth Muscle Cells Induced by TNF- $\alpha$. Chinese Journal of Experimental Traditional Medical Formulae, 17: 159-162.

3. Deng, R.C., Zhou, Y., Zhang, W.S., and Xu, J.G. (2004). Study on mechanism of therapy of vitiligo by Vernonia anthelmintica willd. Letters in Biotechnology, 15: 573-577.

4. Fan, P., Yang, R., and Hua, L. (2009). Curative effect of Tongzhisurunjiang Capsule on 48 gouty arthritis cases. Chinese Jouranl of Traditional Medical Traumotology \& Orthopedics, 17: 43-44.

5. Geng, D.S. (2006). The medical theories and the researching developments of the medicinal herbs in Uygur. Journal of Medicine \& Pharmacy of Chinese Minorities, 13: 5-7.

6. Gu, H.F., Chen, R,Y., Sun, Y.H. and Liu, F. (2004). Studies on chemical constituents from herb of Dracocephalum moldavica. China Journal of Chinese Materia Medica, 29: 232-235.

7. Hui, H.Y., Zhang, C.F., Wu, N., Li, Q.R., and Sun, X.Y. (2010). Effects of Vernonia anthelmintica Willd combined NB-UVB irradiation on the expression of c-KIT and PAR-2 Protein in mice epidermis. Diagnosis and Therapy Journal of Dermato-Venereology, 24: 814-818.

8. Katušin-Ražem, B., Ražem, D., and Dvornik, I. (1983). Radiation treatment of herb tea for the reduction of microbial contamination (Flores chamomillae). Radiation Physics and Chemistry (1977), 22(3): 707-713.

9. Liang, G.Y. (2011). The clinical observation on Kaliziran Tincture combined with Vernohia anthelmintica Willd Injection in vitiligo. China Medical Abstract of Dermatology, 28: 211-212.

10. Liu, Z.J., and Liang, L. (2012). Effect observation of the union treatment with Baixuan Xiatare Tablets and Acitretin Capsules on vulgaris psoriasis. Guide of China Medicine, 10: 197-199.

11. Ma, H.J., Zi, S.X., Li, Y., Liu, W., and Wang, Y.X. (2008). The effect of Vernonia anthelmintica flavone on melanogenesis of human melanocytes in vitro. Chinese Journal of Aesthetic Medicine, 17: 524-526.

12. Nurma-mmat, M., Yang, W.L., Amar, T., and Na-sir, G., (2008). Study on extraction process of total flavonoids in Anchusa italica Retiz. Lishizhen Medicine and Materia Medica Research, 19, 1303-1304.

13. Palida, A., Cong, Y., Ya, M., and Bai, T. (2013). Optimization of ultrasound extraction process of total flavonoids from Anchusa italica by response surface methodology. Chinese Journal of Experimental Traditional Medical Formulae, 19: 48-52.

14. Ren, W.D., Xing, J.G., Wang, X.C., and Xue, G.P. (2011). Preparation and influencing factors on release of total Dracocephalum moldevica flavonoids osmotic pump tablets. Chinese Journal of Experimental Traditional Medical Formulae, 17: 13-17.

15. Song, R., Jin, C.S. and Zhou, Y.W. (2010). To determine content of total flavonoids and Robinin-7-o- $\beta$-D-xylosylglucoside from Dracoephalum mololarie. Chinese Journal of Experimental Traditional Medical Formulae, 16: 71-75

16. Stahl, E. (1969). Thin layer chromatography for characterization of pharmacopeia drugs. 5. Chamomile flowers flores Chamomillae. Arzneimittel-Forschung, 19(11): 1892. 
http://dx.doi.org/10.4314/ajtcam.v11i2.13

17. Tian, Y.Q., Shang, J., He, T., and Cai, W.Y. (2012). Study on material basis of Dracocephalum moldavica for protecting cardiomyocyte against hypoxia/reoxygenation injury by traditional Chinese medicine serum chemical and pharmacological methods. China Journal of Chinese Materia Medica, 37: 620-623.

18. Tang, Z.L., Peng, Y.N., Shao, C.G. and Zhou, X.L. (1998). A correlation study of incidence of psoriasis and meteorological factors. Chinese Journal of Dermatology, 31: 75-77.

19. Upur, H., Yusup, A., Baudrimont, I., Umar, A., Berke, B., Yimit, D. and Lapham,, J. C. (2011). Inhibition of cell growth and cellular protein, DNA and RNA synthesis in Human Hepatoma (HepG2) cells by ethanol extract of Abnormal Savda Munziq of traditional Uighur medicine. Evidence Based Complementary and Alternative Medicine, 25:14-24.

20. Wang, J.T. (2012). Effect observation of the union treatment with NB-UVB and Baixuan Xiatare tablet on vulgaris psoriasis. Diagnosis and Therapy Journal of Dermato-Venereology, 19: 294-297.

21. Wang, Y.Z., Lv, F.M. and Han, Y.M. (2005). Introduction of Uighur medicine's resource and theory. China Journal of Chinese Materia Medica, 30: 316-317.

22. Wei, K.K., Hu, B.S. and Gao, Y.J. (2009). The effect observation on Fufang Kaliziran Tincture combined with Vernohia anthelmintica Willd Injection in vitiligo. Journal of Medical Forum, 30: 94-95.

23. Wen, Q., Chen, J.Y., and Liu, H.X. (2013). Geographical feature and treatment strategy of psoriasis's formation. Xinjiang Journal of Traditional Chinese Medicine, 31: 3-4.

24. Wu, T., Jiang, L., Aisa, H. and Sun, Y.H. (2011). Study on new extraction technology and protective effect of Hugan Buzure Granules on liver injury in mice. China Journal of Chinese Materia Medica, 36: 429-433.

25. Xu, Z.W. (1981). The research on the chemical component of Anchusa. China Journal of Chinese Materia Medica, 2: 79-80.

26. Yan, L.L., Kang, X.L. and He, C.H. (2013). Effects of moldavica total flavone on regulation of Th1/Th2 imbalance in ovalbumin-induced asthmatic rats. Journal of China Medical University, 42:142-145.

27. Yang, L.N., Xing, J.G., He, C.H. and Yang, P.M. (2013). Chemical constituents of Dracocephalum moldavica L. and their pharmacological Activities. World Clinical Drugs, 34(4): 226-231.

28. Yao, L. (2007). Experimental study of the correlation between different extraction methods and functional mechanisms of Vernonia anthelmintica. Master Thesis at Xinjiang Medical University.

29. Yao, Y.T. (2012). The tablet of Tongzhisurunjian study on the Preparation and quality standard. Master Thesis at Wuhan University of Technology.

30. Yuan, Y., Long, Z.J., Yang, J.J. and Yuan, C.H. (2011). Study on the anti-inflammatory effect of volatile oil from Matricaria Chamomilla L. Pharmaceutical Biotechnology, 18: 52-55.

31. Yu, L.H., Sun, L., Li, Y.J., and Shang, J. (2007). Study on extraction techniques for Vernonia anthelmintica by grading methods. Chinese Journal of Modern Applied Pharmacy, 5:373-375.

32. Zhao J., Luo Z., and Wu S. (2004). In vitro anti-candidal activity of the essential oil of Illicium verum. Chinese Journal of Dermatology, 37(8): 475-477.

33. Zhou, X.Y., Hu, X.J., Tian,S.G., Zhou, Z.C. (2000). Research on Chemical Component of the Fruit and Various Medical Forms of Uigurian Medicine Fructus Vernonia. Chinese Journal of Ethnomedicine and Ethnopharmacy, 2: 023.

34. Zhu, L., Ying, W. and Yi, Z. (2013). The effect of Tongzhisurunjiang Capsules to treat gouty arthritis. Asia-Pacific Traditional Medicine, 9: 171-172. 